\title{
Rise of Central China Strategy and the Economic Growth of Hubei Province
}

\author{
Pengfei Wang \\ Institute of Economy, Jinan University, Guangzhou, China \\ Email:2572480335@qq.com
}

How to cite this paper: Wang, P.F. (2019) Rise of Central China Strategy and the Economic Growth of Hubei Province. Modern Economy, 10, 502-512. https://doi.org/10.4236/me.2019.102034

Received: January 18, 2019

Accepted: February 16, 2019

Published: February 19, 2019

Copyright $\odot 2019$ by author(s) and Scientific Research Publishing Inc. This work is licensed under the Creative Commons Attribution International License (CC BY 4.0).

http://creativecommons.org/licenses/by/4.0/

\begin{abstract}
In order to verify the economic effect of the strategy of the rise of the central region, based on the panel data of 26 provinces in China from 1994 to 2016, taking Hubei Province as an example, the author uses the synthetic control method to analyze the impact of the strategy of the rise of the central region on the economic growth of Hubei Province. The study shows that the strategy of the rise of the central region has significantly promoted the economic growth of Hubei Province, which is mainly achieved by improving the investment environment and improving the level of education.
\end{abstract}

\section{Keywords}

Rising Strategy for Central China, Economic Growth Performance, Synthetic Control Method

\section{Introduction}

In order to coordinate regional economic development and promote economic growth in central China, the central government launched the strategy of central China's rise in 2006. In more than a decade, the country implemented a number of measures to ensure the execution of the strategy of central China's rise. For example, the government promulgated some guidance documents, supported the construction of the urban agglomeration like Taiyuan, Zhengzhou, Wuhan and Changsha, held Central China investment and trade fair and so on. So how effective are these measures on economic growth? The analysis and discussion of this question is helpful to the implementation and improvement of the follow-up measures. Taking Hubei province as an example, this paper analyzes the economic effect of the strategy of central China's rise on Hubei province by using the synthetic control method, and finds that the strategy effectively promotes the economic growth of Hubei province, and mainly promotes the economic growth 
by improving the investment environment and improving the education level. The main contribution of this paper lies in the introduction of synthetic control method for the first time to analyze the economic effect of the strategy of central China's rise and further analysis of the mechanism of the effect.

\section{Research}

At present, there are few literatures on the economy performance evaluation of the strategy of central China's rise. Ji Xinfeng used the 1997-2009 provincial panel data, and used the systematic generalized moment estimation method and the quadratic difference method to evaluate the strategy of central China's rise [1]. He found that the implementation of the strategy of central China's rise did not accelerate the economic growth of the central region. On the contrary, li Hongyan and Wang Tao used panel data of six provinces in central China from 2002 to 2006 for regression analysis and found that the rise effect of central China has been shown [2]. By comparing the relevant economic indicators before and after the implementation of the rise strategy of the central region, Yu Xinan et al. found that the rise strategy of the central region has achieved remarkable results in economic strength, structural transformation, people's living standards and other aspects [3]. We can find that these literatures either evaluate the strategy of central China's rise at the very beginning of its implementation, or evaluate it by simple comparison of indicators without distinguishing other factors that may affect economic growth.

Comparatively speaking, there are many literatures analyzing the strategic economic performance of western development, and the methods used are more scientific and reasonable. Liu Shenglong et al. studied the economic impact of the western development strategy on the western region by using the dual difference method (DID) [4]. Liu Ruiming et al. further estimated the net effect of the western development strategy on economic growth in the western region by using the psm-did method [5]. However, both methods have some disadvantages. The basic idea of the dual difference method is: selecting a treatment group and a control group, the treatment group and control group were not affected by the policy before some point, and after this time, policy hits the treatment group, but policy do not hit the control group, so researchers can estimate the effect of the policy on the treatment group. This method requires that the factors determining economic growth of the control group and the treatment group are the same, and these determinants have the same degree of influence on the control group and the treatment group. In reality, finding a control group that meets these criteria is not easy. First, policies tend to be built into the treatment group. Why does the treatment group produce such a policy when the control group does not? It is precisely because there are some factors in the treatment group that lead to policies, and those factors do not exist in the control group, there will be no policies in the control group. This means that the great western development strategy will only be implemented in the western region, and not out- 
side the western region. The above analysis shows that the treatment group is different from the control group. Secondly, when using the dual difference method, the selection of the control group is usually based on the researcher's preference and experience. The control group chosen by different researchers is often different, resulting in different conclusions. The psm-did method further improved the selection of the control group and selected the control group most similar to the economic growth determinants of the treatment group through the weighted average method of propensity score matching. However, this method cannot guarantee that these factors have the same influence on the treatment group and the control group, and this method requires a large sample size.

The synthetic control method [6] adopted in this paper can effectively solve the above problems. The basic idea of synthetic control method is as follows: since it is difficult for us to find satisfactory control group in reality, we might as well construct a "counterfactual" control group: through the weighted average of the control group outside the treatment group. Specifically, if we want to observe the impact of the rise strategy of central China on the per capita GDP of Hubei province, we can construct the "counterfactual" Hubei province as the control group by weighting the average of other provinces outside the six central provinces. As long as we can guarantee prior to the implementation of the strategy of central China's rise the per capita GDP of "counterfactual" Hubei province is consistent with the per capita GDP of Hubei province, then we can observe the influence of the strategy of central China's rise through comparing the per capita GDP after the implementation of the strategy of central China's rise. This approach allows economic growth determinants to have varying degrees of influence on the treatment and control groups. Because when constructing the "counterfactual" control group, we require that the economic growth trend of the "counterfactual" control group and the treatment group should be consistent. Even if there are differences between some regions of the control group and the treatment group, these differences will be weighted out in the construction process. In addition, when constructing the "counterfactual" control group, the weight assigned to the control group is determined by the data, which avoids the subjective choice of researchers. The contribution of this paper is mainly reflected in two aspects: first, this paper introduces the synthetic control method to evaluate the net effect of regional development strategy on regional economic growth. This method has a series of advantages over DID and PSM-DID methods, and can effectively control the selectivity bias and endogenous problems that can't be solved by DID and psm-did methods. Secondly, the main difference between this paper and the domestic literature using synthetic control method is that the author not only examines the economic growth effect of policies, but also examines the sustainability of policy impact and its causes, which can provide policy reference for the further deepening of regional development strategy. Therefore, this paper chooses the synthetic control method to evaluate the economic effect of the rise strategy of central China. 


\section{Empirical Analysis}

\subsection{Variable and Data}

The question to be discussed in this paper is whether the rise strategy of central China has promoted the economic growth of Hubei province. Therefore, the outcome variable is set as the economic growth, which is measured by the per capita GDP. Refer to previous studies, the main factors (predict variables) in this paper that will affect the outcome variables is set as primary industry output value proportion of GDP, the second industry output value proportion of GDP, tertiary industry output value proportion of GDP, government size (measured by the general budget spending as a share of GDP) and foreign direct investment level (measured by the actual use of foreign direct investment as a share of GDP), the level of investment in fixed assets (measured by the whole society fixed asset investment as a share of GDP), education level (using the proportion of college students in the total population).

This article USES the 1994-2016 (with the exception of Hong Kong, Macao and Taiwan) of China in 26 provinces, municipalities and autonomous regions balance panel data to evaluate the economic effects of the strategy of central China's rise. Hubei province which has implemented the strategy of central China's rise since 2006 is set as treatment group, the rest of the 25 provinces, municipalities and autonomous regions is set as the control group, including Beijing, Shanghai, Tianjin, Hebei, Jiangsu, Zhejiang, Fujian, Shandong, Guangdong, Hainan, Liaoning, Jilin, Heilongjiang, Inner Mongolia, Guangxi, Chongqing, Sichuan, Guizhou, Yunnan, Tibet, Shaanxi, Gansu, Qinghai, Ningxia and Xinjiang. The data of variables before 2008 were mainly from the compilation of statistical data of the People's Republic of China in the past 65 years. The data after 2008 were mainly from the statistical yearbook of China. Some missing data were supplemented from the statistical yearbooks of corresponding provinces, cities and autonomous regions in the corresponding years. Descriptive statistics of each variable are shown in Table 1.

\subsection{Result}

This part of the empirical analysis is as follows: first, the use of synthetic control method to test whether the implementation of the rise strategy of central China has promoted the economic growth of Hubei province. Second, the placebo method was used to test the effectiveness of the rise strategy of central China on economic growth. Finally, the significance of the rising strategy of central China on economic growth is tested by permutation test.

In fact, the synthetic control method gives different weights to the control group and constructs "counterfactual" Hubei province. Then the "counterfactual" Hubei province is compared with real Hubei province. Table 2 shows the weight of provinces in the control group, among which 8 provinces with positive weight are Jilin, Xinjian, Tibet, Sichuan, Hebei, Gansu, Heilongjiang and Fujian from the largest to the smallest. 
Table 1. Descriptive statistics of major variables.

\begin{tabular}{ccccc}
\hline Variate & Maximum & Minimum & Average & SD \\
\hline Per capita GDP & 11.6801 & 7.3311 & 9.6455 & 0.9854 \\
Proportion of primary industry & 45.9700 & 0.3900 & 14.6321 & 8.3765 \\
Proportion of secondary industry & 60.1300 & 17.1300 & 44.0872 & 8.6606 \\
Proportion of tertiary industry & 80.2300 & 27.6700 & 41.2774 & 5.3308 \\
Fixed asset investment level & 138.6200 & 23.9800 & 23.2155 & 17.2393 \\
The size of government & 137.9200 & 4.9200 & 20.3370 & 3.4532 \\
Level of foreign direct investment & 24.2543 & 0.0014 & 3.2518 & 1.1834 \\
Education level & 3.9900 & 0.0900 & 0.8569 \\
\hline
\end{tabular}

Table 2. The weight of control group.

\begin{tabular}{ccccccccccc}
\hline Province & Weight & Province & Weight & Province & Weight & Province & Weight & Province & Weight \\
\hline Beijing & 0 & Zhejiang & 0 & Liaoning & 0 & Chongqing & 0 & Shaanxi & 0 \\
Tianjin & 0 & Fujian & 0.003 & Jilin & 0.196 & Sichuan & 0.135 & Gansu & 0.115 \\
Hebei & 0.134 & Shandong & 0 & Heilongjiang & 0.110 & Guizhou & 0 & Qinghai & 0 \\
Shanghai & 0 & Guangdong & 0 & Inner Mongolia & 0 & Yunnan & 0 & Ningxia & 0 \\
Jiangsu & 0 & Hainan & 0 & Guangxi & 0 & Tibet & 0.140 & Xinjiang & 0.167 \\
\hline
\end{tabular}

The synthetic control method requires that the synthetic Hubei province can well fit the Hubei province before the implementation of the rise strategy of central China. It is embodied in the fact that the synthetic Hubei province is close to the real Hubei province in the key index reflecting the economic situation. Table 3 shows the comparison between real Hubei and synthetic Hubei's key economic indicators before the implementation of the rise strategy of central China. We can find that the synthetic Hubei province is similar to the real Hubei province, and the difference between the two is relatively small, which indicates that the synthetic Hubei province fits the Hubei province before the implementation of the central rise strategy better, so we can compare the difference between the two after the implementation of the central rise strategy and observe the economic effect of the central rise strategy.

Figure 1 depicts the development path of real and synthetic logarithms of GDP per capita in Hubei province. We can find that, before the implementation of the rise strategy of central China, the routes of the synthetic Hubei province and the real Hubei province basically coincide completely, which further shows that the synthetic Hubei province can well fit the real Hubei province. After the implementation of the strategy of the rise of central China, the logarithm of real per capita GDP of Hubei province exceeded that of synthetic Hubei province, and the gap between the two is increasing with the passage of time. This shows that the implementation of the strategy of the rise of central China has promoted the economic development of Hubei province. 
Table 3. The comparison table of predictive variables.

\begin{tabular}{ccc}
\hline Variable & The real Hubei & The synthetic Hubei \\
\hline Proportion of primary industry & 17.82 & 18.47 \\
Proportion of secondary industry & 43.04 & 42.59 \\
Proportion of tertiary industry & 39.14 & 38.94 \\
Fixed asset investment level & 53.33 & 58.70 \\
The size of government & 13.22 & 28.03 \\
Level of foreign direct investment & 2.71 & 1.32 \\
Education level & 1.45 & 0.99 \\
\hline
\end{tabular}

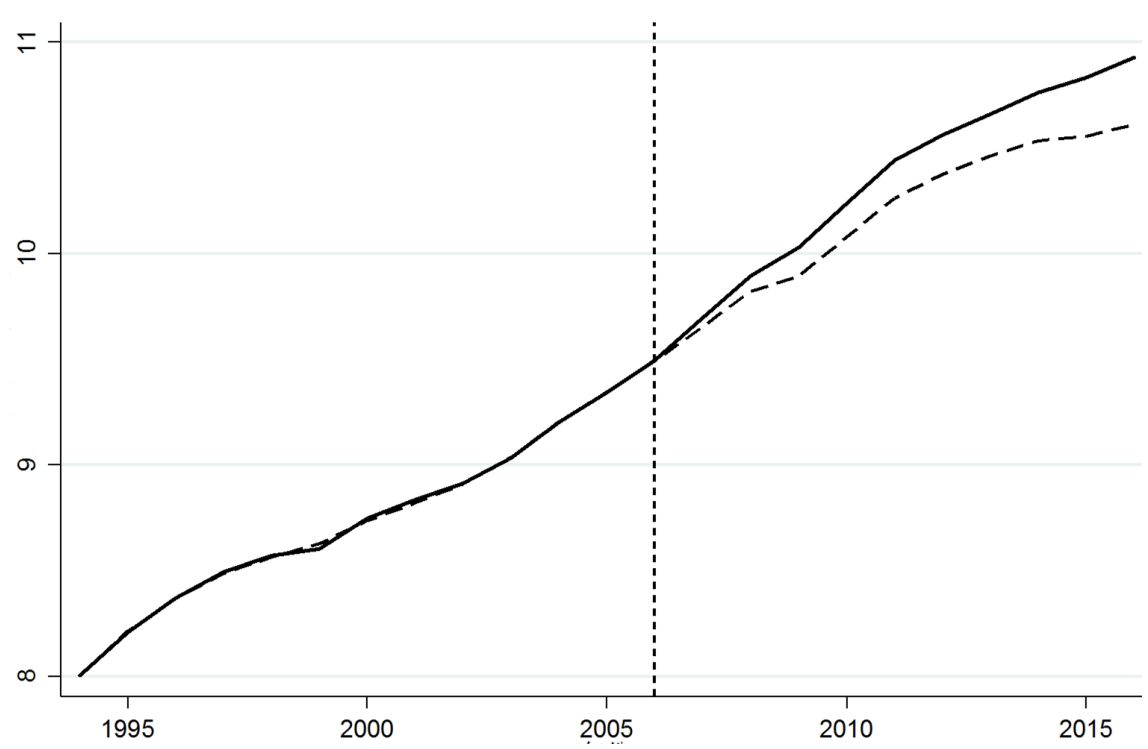

Figure 1. The contrast between the real Hubei and the synthetic Hubei.

The author further calculates the logarithmic growth rate of real and synthetic Hubei per capita GDP before and after the implementation of the strategy of central China's rise. Before the implementation of the strategy of the rise of central China, i.e. from 1994 to 2005, the logarithmic growth rates of real Hubei province and synthetic Hubei province were $1.4195 \%$ and $1.4191 \%$ respectively, with little difference between the two. After the implementation of the strategy, namely from 2006 to 2016, the logarithmic growth rate of real and synthetic Hubei province was $1.4343 \%$ and $1.1603 \%$ respectively, and the difference between the two was $0.274 \%$, which was a considerable growth effect. In addition, the author also calculated the difference between the logarithm of real per capita GDP of Hubei province and the synthetic Hubei province, and drew it into Figure 2, so that we can more intuitively see the economic effect of the rise strategy of central China. As can be seen from Figure 2, before the implementation of the rise strategy of central China, the difference between the two was within 0.01 . After the implementation of the strategy, the gap between the two gradually widened, reaching 0.31 in 2016. 


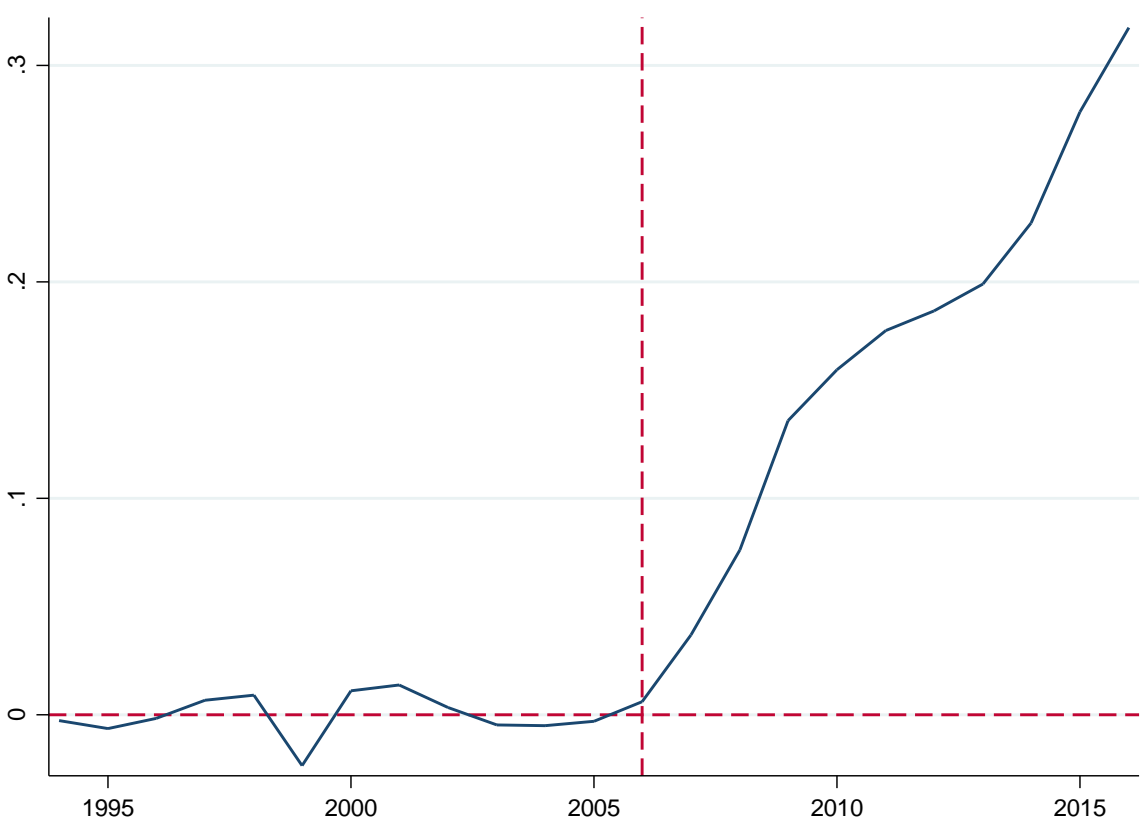

Figure 2. The gap of per capita GDP.

Although the above analysis shows that the rise strategy of central China has promoted the development of Hubei province, is it an accidental phenomenon? Is it not the result of strategy implementation? In order to enhance the effectiveness of the research conclusions, the placebo method will be used in this paper. Placebo method is from medical experiments: if you want to examine the effects of a new drug, the patients will be randomly divided into two groups, one group of taking drugs, another group of taking placebo (such as sugar pill), and the research don't tell two groups of people taking the drug or placebo in order to avoid the interference of psychological factors, and then by looking at the condition of the two groups of people to examine the effects of new drugs. In this paper, the basic idea of placebo method is: since 25 provinces outside of Hubei province did not implement the strategy of rise of central China, then choose a province in 25 provinces, assuming that the province implements the strategy of rise of central China in 2006, we use synthetic control method to analyses this province, just like the analysis of Hubei province. The province's per capita GDP should be similar to the per capita GDP of synthetic province, or synthetic control analysis of Hubei province is only an accidental phenomenon, has no credibility. Referring to the practices of previous researchers [7], this paper selected Jilin province, which has the largest weight in the composition and synthesis of Hubei province, as the placebo. Figure 3 depicts the development path of the logarithm of real and synthetic per capita GDP of Jilin province. We can find that, before 2006, the development paths of the logarithm of real per capita GDP of Jilin province and synthetic per capita GDP of Jilin province basically coincide, indicating that the synthetic Jilin province can well fit the economic development of real Jilin province. From 2006 to 2013, the per capita GDP of real Jilin province was higher than that of synthetic Jilin province; from 


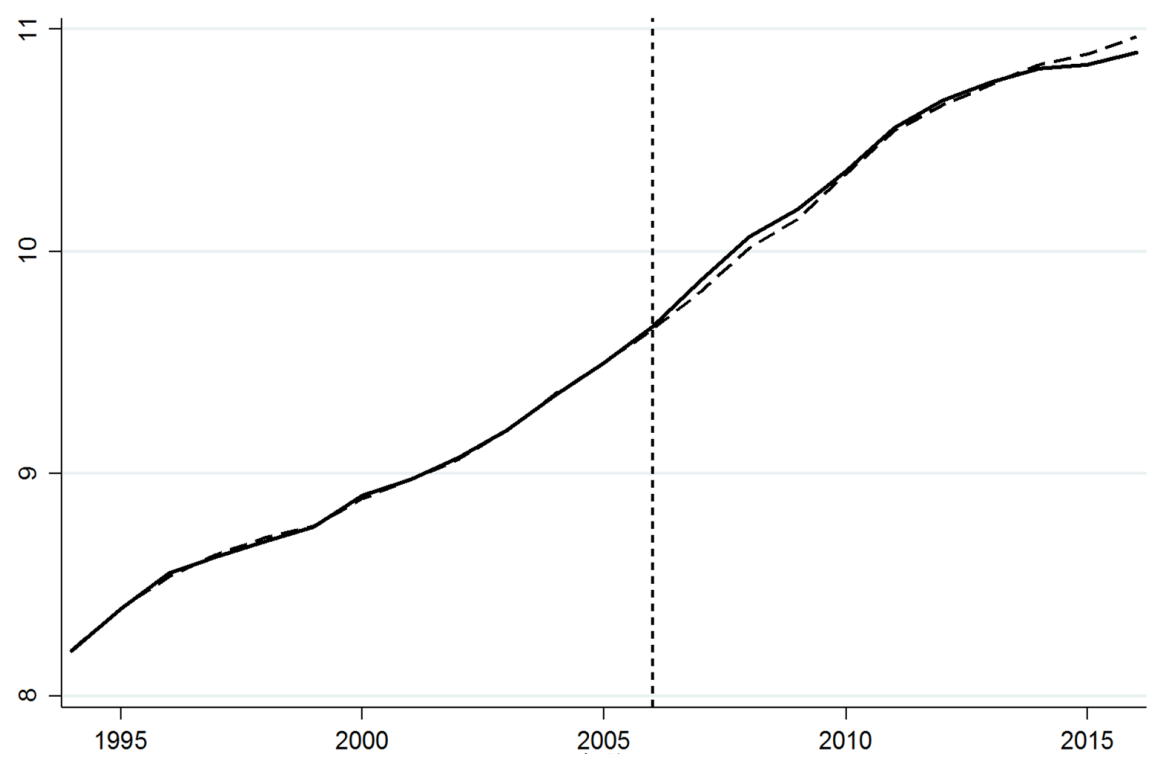

Figure 3. The contrast between the real Jilin and the synthetic Jilin.

2013 to 2016, the synthetic Jilin province is higher than the real Jilin province, which is consistent with the observation conclusion of Yang Tianfu and Rong Yuwei [8] on the strategy of northeast rejuvenation. However, the difference between synthetic Jilin province and real Jilin province is not as great as that between synthetic Hubei province and real Hubei province. The placebo test proved the effectiveness of the rising central China strategy in promoting economic growth in Hubei province.

The significance level of the policy effect is often shown in the policy evaluation by using the double difference method and other methods. So how do you represent the level of significance of policy effects in synthetic control? Arrange inspection method is proposed to check whether the policy effect of significance in the statistical significance by Abadie [9]. Its specific ideas in this article are: taking placebo inspection for all the provinces in the control group, and calculating the difference of the real provinces and the synthetic provinces in per capita GDP, if the rise of central China strategy effect to the economy of Hubei province is true, then the difference between real and synthetic in Hubei province, Hubei province in all 26 provinces should is the biggest of all. Figure 4 shows the logarithmic difference of GDP per capita in 26 provinces. We can find that the difference between the real and synthetic logarithm of GDP per capita in Hubei province is greater than that of most provinces.

\subsection{Mechanism Analysis}

What is the reason that the rise strategy of central China has promoted the economic growth of Hubei province? From the perspective of Liu Ruiming and Zhao Renjie, this paper also identifies the causes by examining the effects of the rise strategy of the central region on various economic growth factors. But this article is different from what they did, their way is: set the region, policy, two 


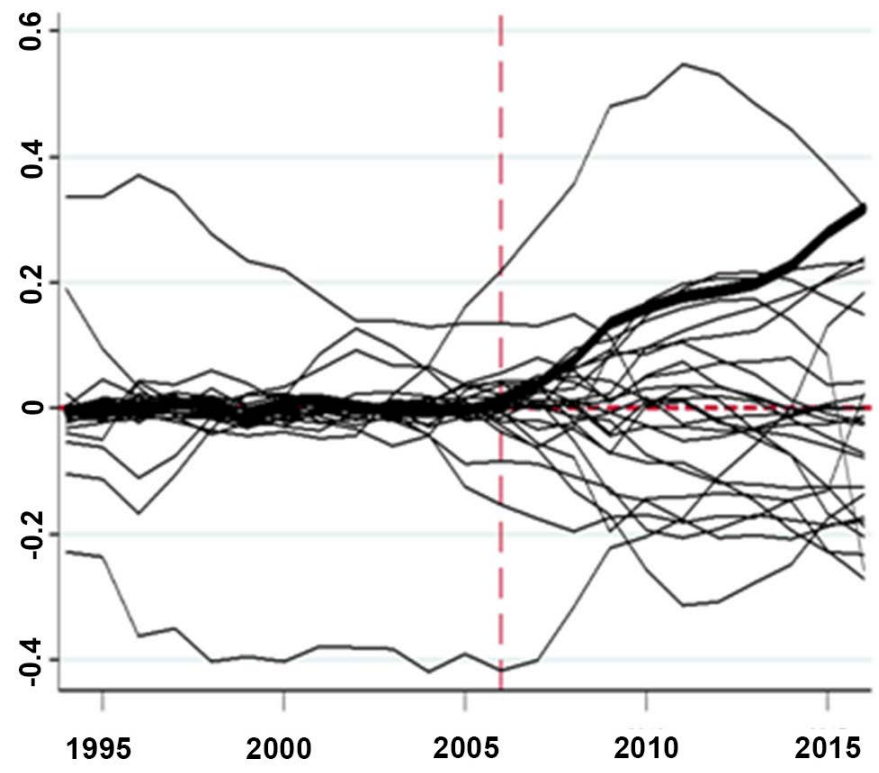

Figure 4. The logarithmic difference of GDP per capita in 26 provinces.

virtual variables, then region virtual variables, policy variables and interaction of virtual variables and policy variables as explanatory variables, the various factors that affect economic growth as the explained variable regression analysis, interaction item represents the policy on the various factors affecting economic growth, the net effect. The approach of this paper is to continue to use the synthetic control method to analyze the net effect of the rising central China strategy on various economic growth factors. Since synthetic control method can better identify the impact of strategy implementation on the numerical value of per capita GDP than other methods, it is natural to better identify the impact of strategy implementation on other factors. In this particular way is: to set the various influence factors of economic growth as the variables, including industrial structure (measure) by the second industry accounted for the proportion of the tertiary industry, the level of investment in fixed assets, the government scale, foreign direct investment level and education level, and then use other fitting the data of 25 provinces, respectively to calculate the strategy implementation after the real value of average annual growth, synthetic value of average annual growth rate, average annual growth rate and the synthetic value of the real value of the average annual growth rate of difference, the rise of central China strategy by analyzing the difference we can observation on the influence of various factors.

Looking at Table 4, we can find that the real value of the logarithmic value of per capita GDP is 0.274 percentage points higher than the composite value, which indicates that the rising strategy of central China promotes the economic growth of Hubei province. Taking 0.274 as the dividing line, we can divide the influence of the rise strategy of central China on various economic growth factors into two categories. The first category is higher than 0.274 , such as the influence of the strategy on the level of foreign direct investment and the education 
Table 4. The influence of the rising strategy on various economic growth factors (\%).

\begin{tabular}{cccc}
\hline Variable & The real value & The composite value & Gap \\
\hline Per capita GDP & 1.434 & 1.160 & 0.274 \\
The industrial structure & 0.492 & -0.675 & -2.323 \\
Fixed asset investment level & 7.147 & 7.082 & 0.064 \\
The size of government & 4.834 & 4.756 & 0.078 \\
Level of foreign direct investment & -2.235 & -6.932 & 4.697 \\
Education level & 3.291 & 2.263 & 1.028 \\
\hline
\end{tabular}

level. The other category is less than 0.274 , such as the impact on the industrial structure, the level of fixed asset investment, the impact on the size of the government. The level of foreign direct investment reflects the "soft environment" strength of a region's future economic development potential, and can comprehensively reflect the investment environment of a region, such as the efficiency of the government of the region, the level of government services, the openness of the region, the state of infrastructure and institutional conditions. Education level reflects a region's human capital level, and the classic literature [10] shows the importance of human capital level to economic growth. It can be seen that the implementation of the strategy of rising central China has improved the economic development conditions of Hubei province and laid a good foundation for the long-term economic development. The difference between the real value and the synthetic value of fixed asset investment level and government scale is not large, which indicates that the economic growth of Hubei province mainly comes from the improvement of foreign direct investment level and education level. The real value of the industrial structure level is positive, and the composite value is negative, which means that the development of the secondary industry in Hubei province is faster than that of the tertiary industry. This change may be because the central region receives a large number of industries from the eastern region.

\section{Conclusion}

In this paper, the net effect of the rising central region strategy on the economic growth of Hubei province is tested for the first time by using the synthetic control method, and the mechanism of the rising central region strategy is analyzed. The results showed that the strategy effectively promotes the economic growth of Hubei province, and mainly promotes the economic growth by improving the investment environment and improving the education level. The limitation of this paper lies in that it does not examine the economic effect of the rising strategy of central China from the perspective of the six provinces of central China, which will be the follow-up work of the author.

\section{Conflicts of Interest}

The author declares no conflicts of interest regarding the publication of this paper. 


\section{References}

[1] Ji, X.F. (2012) An Analysis of the Strategy of Promoting the Rise of Central China and the Regional Economic Gap in China. Future and Development, 35, 89-94.

[2] Li, H.Y. and Wang, T. (2007) An Empirical Study on the Rising Effect of Central China. Statistics and Decision-Making, No. 21, 48-49.

[3] Yu, X.N., Yang, L.Q., Liu, X.P. and Guo, Z.Y. (2014) Effectiveness, Experience and Future Orientation of the Strategy of Rising Central China in the Ten Years. Academic Journal of Zhongzhou, No. 9, 45-54.

[4] Liu, S.L., Wang, Y.H. and Hu, A.G. (2009) Effectiveness of Western Development and Regional Economic Convergence in China. Economic Research, 44, 94-105.

[5] Liu, R.M. and Zhao, R.J. (2015) Western Development: Growth Driven or Policy Trap: A Study Based on PSM-DID Method. China Industrial Economics, No. 6, $32-43$.

[6] Abadie, A. and Javier, G. (2003) The Economic Costs of Conflict: A Case Study of the Basque Country. American Economic Review, 93, 113-132. https://doi.org/10.1257/000282803321455188

[7] Wang, X.B. and Nie, H.F. (2010) Administrative Division Adjustment and Economic Growth. Managing the World, No. 4, 42-53.

[8] Yang, T.Y. and Rong, Y.F. (2017) Can Regional Development Strategy Promote Economic Growth: A Case Study of Revitalizing the Old Industrial Base in Northeast China. Economic Theory and Economic Management, No. 10, 88-99.

[9] Abadie, A., Diamond, A. and Hainmueller, J. (2010) Synthetic Control Methods for Comparative Case Studies: Estimating the Effect of California's Tobacco Control Program. Journal of the American Statistical Association, 105, 493-505. https://doi.org/10.1198/jasa.2009.ap08746

[10] Schultz, W. (1961) Investment in Human Capital. American Economic Review, 51, 1-17. 